\title{
An unexpected complication after EGD
}

\author{
Daniel Hernandez MD, Marcella Rivas MD, David Sotello MD, Ebtesam Islam MD, PhD
}

\begin{abstract}
An eighty-two-year-old woman with a history of carotid atherosclerosis, peptic ulcer disease, hypertension, and right knee osteoarthritis was admitted to the hospital for observation after an uncomplicated left total knee arthroplasty. Her home medication clopidogrel was continued while in the hospital, and she received ketorolac as needed for pain. The night after surgery she developed coffee ground emesis and hematochezia associated with tachycardia and hypotension that did not respond to resuscitation with intravenous fluids. The patient was transferred to the medical intensive care unit and started on norepinephrine, intravenous proton pump inhibitors, and octreotide. Gastroenterology was consulted, and esophagogastroduodenoscopy (EGD) was performed. During the procedure, the patient developed new onset abdominal distention, and the procedure was terminated. Her chest x-ray showed sub-diaphragmatic gas under the right hemi-diaphragm, and general surgery was consulted (Figure 1). The patient underwent emergent laparotomy, and a one $\mathrm{cm}$ perforation in the anterior body of the stomach was repaired. After laparotomy the patient remained critically ill. An EGD was repeated two days later and showed multiple non-bleeding gastric ulcers and two duodenal ulcers with possible exposure of a gastroduodenal artery. Due to persistent melena, embolization of gastroduodenal artery was performed. The patient remained critically ill on mechanical ventilation, and developed acute renal failure requiring continuous renal replacement therapy. Despite maximal therapy she died several days later.
\end{abstract}

\section{Discussion}

Pneumoperitoneum refers to the presence of air in the peritoneal cavity. Most cases of pneumoperitoneum

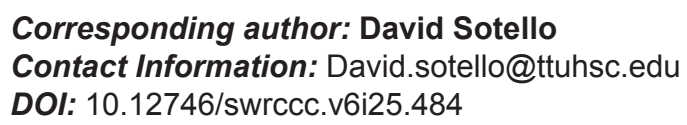

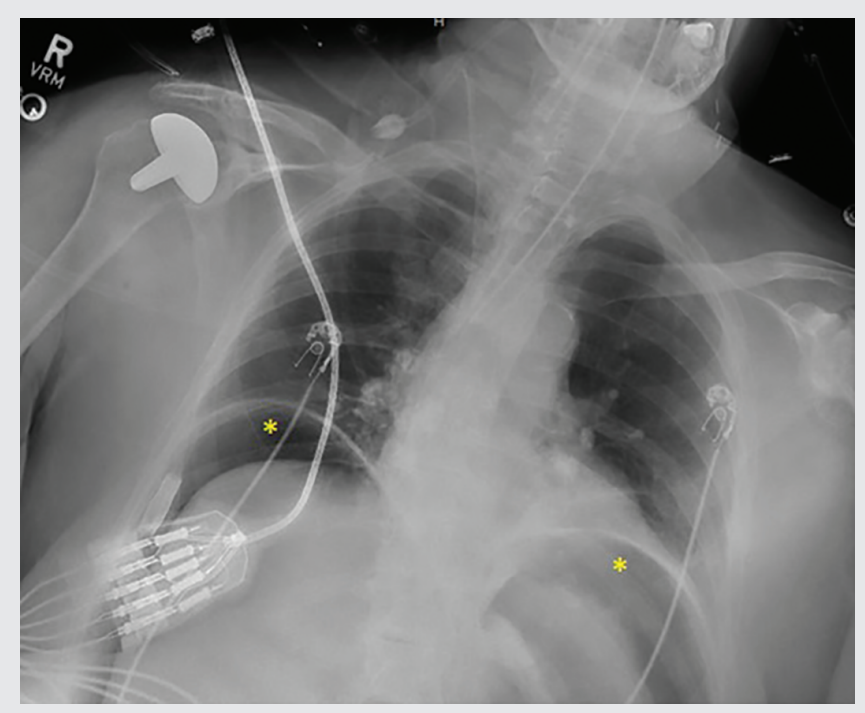

Figure 1. AP portable chest x-ray showing subdiaphragmatic air under the diaphragms $(*)$.

result from a perforated viscus which is a surgical emergency, but it can also occur as an expected finding in other clinical situations, including after abdominal surgery, after placement and manipulation of peritoneal dialysis catheters, after placement of a percutaneous endoscopic gastrostomy, or after polypectomy during colonoscopy, etc. ${ }^{1}$

Viscus perforation occurs as a complication of EGDs in $0.02 \%$ to $0.2 \%$ of cases. The most common sites of perforation during an EGD are the proximal esophagus, anatomical lead points, and at any location of the GI tract with abnormal anatomical features, such as inflammation, neoplasms, dysplasia, or acute injury. ${ }^{2}$ Peptic ulcers can also develop perforations. This complication has a high mortality rate, and as many as $37 \%$ of all deaths related to ulcers are attributed to perforation, which is 5 -fold higher than deaths attributed to bleeding. ${ }^{3}$ Our patient's risk for complications was increased with the concurrent use of antiplatelet and non-steroidal anti-inflammatory drugs. 


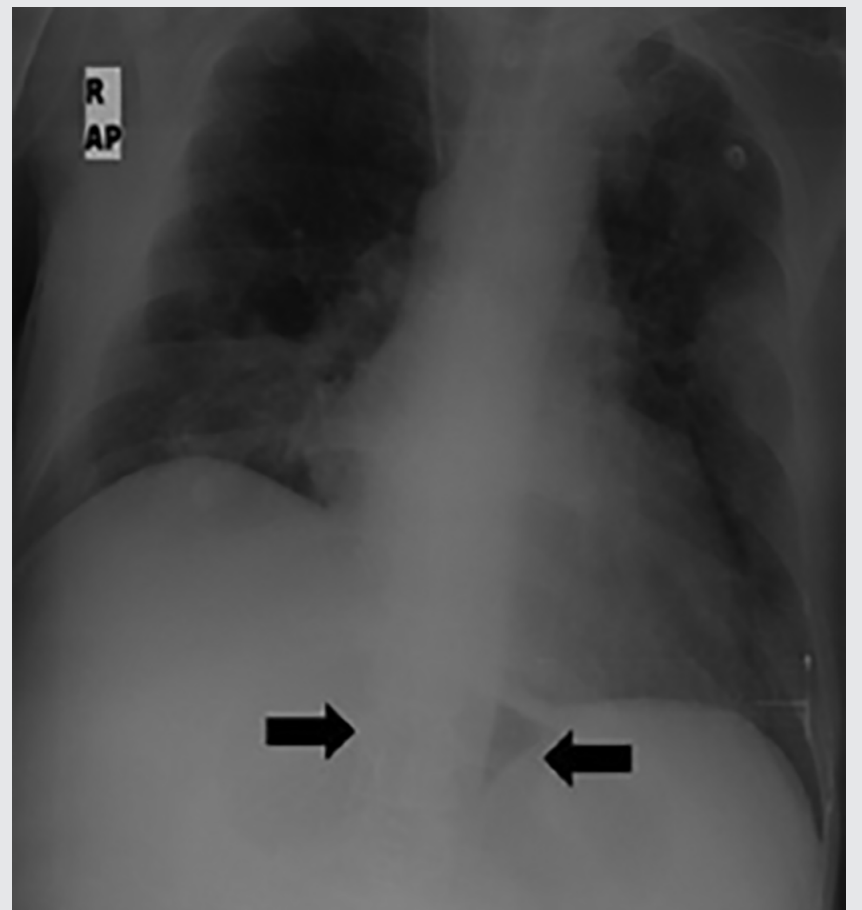

Figure 2. Positive cupola sign (black arrows). ${ }^{8}$

Imaging studies can help identify air inside the abdominal cavity when clinically suspected. Abdominal computed tomography (CT) scans have been considered the gold standard for the diagnosis of pneumoperitoneum. However, its sensitivity and specificity are similar to other imaging studies, such as erect frontal and/or lateral chest $x$-rays, supine full abdominal $\mathrm{x}$-rays, or abdominal sonograms. X-rays and CT scans can identify as little as 1-2 $\mathrm{mL}$ of air in the abdominal cavity while a sonogram identifies $\geq 2 \mathrm{~mL}{ }^{3,4,5} \mathrm{On}$ chest $\mathrm{x}$-rays, the presence of subdiaphragmatic air and a positive cupola sign, which refers to an arcuate lucency overlying the lower thoracic spine projecting towards the heart, are both diagnostic of pneumoperitoneum (Figure 2). ${ }^{6}$ On abdominal x-rays, the right upper quadrant gas sign is highly sensitive to detect pneumoperitoneum and refers to the presence of air in the right upper quadrant of the abdominal cavity (Figure 3). Other less sensitive signs include Rigler's sign (Figure 4) which is described as a bowel wall enhancement by intraluminal and extraluminal air, the

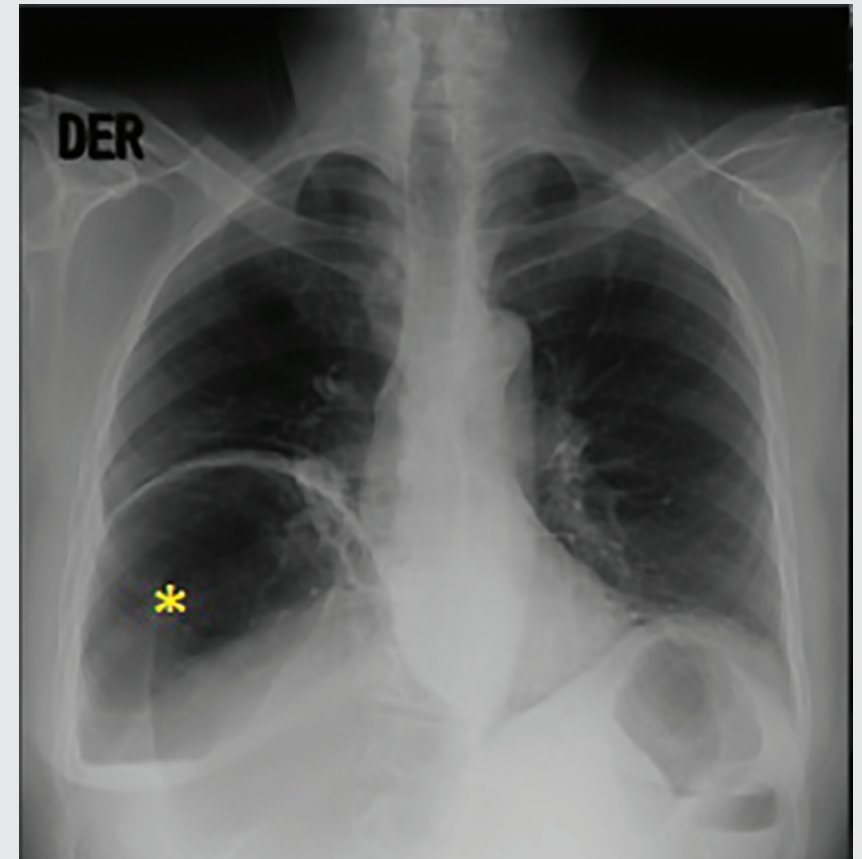

Figure 3. Right upper quadrant sign $(*) .{ }^{9}$

falciform ligament sign in which the falciform ligament becomes visible due to the free air, and the inverted $\mathrm{V}$ sign which refers to enhancement of the lateral umbilical ligaments due to free air.

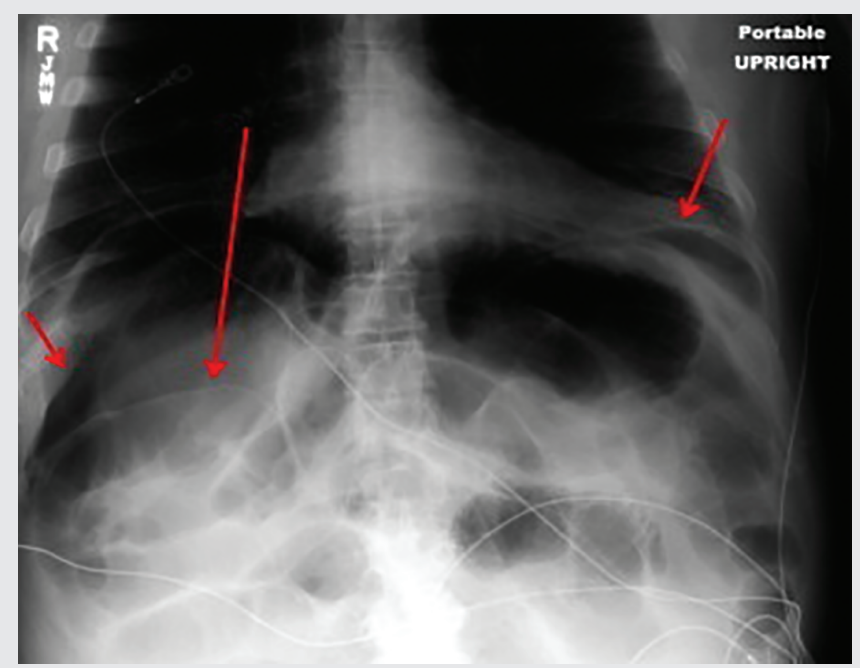

Figure 4. Positive Rigler's sign (red arrows). ${ }^{10}$ 
The accuracy of abdominal sonograms to detect pneumoperitoneum is highly dependent on the technician's expertise since differentiating free air from air in the lungs or in a hollow viscus can be challenging. Sonographic signs of free peritoneal air include gas above the liver, which results in a brighter liver capsule and an obscured liver parenchyma due to free gas, the movable gas sign, which refers to repositioning of the gas with patient motion or while applying pressure with the transducer, and, finally, peritoneal line enhancement, another sign that refers to a continuous or adjacent brightness of the peritoneal line due to the reflecting quality of the air in the sonogram. ${ }^{5}$

Keywords: pneumoperitoneum, peptic ulcer, esophagogastroduodenoscopy

Figures-Figures 2,3 and 4 were obtained from OPENi (beta), an open source for medical images, at the Texas Tech University Health Sciences Center Library in Lubbock, Texas. Relevant references are 8,9, and 10. Accessed 6/28/2018.

From: Department of Internal Medicine at Texas Tech University Health Sciences Center in Lubbock, Texas, and Universidad Dr Jose Matias Delgado, El Salvador (DH).

Submitted: 5/23/2018

Reviewer: Kenneth Nugent MD

Conflicts of interest: none

This work is licensed under a Creative Commons

Attribution-ShareAlike 4.0 International License

\section{REFERENCES}

1. Howe C. Pneumoperitoneum: What does free air under the diaphragm really mean in the older adult? Ariz Geriatr Soc J 2007; 13:23-24.

2. Wolfsen H. Complications of Esophagogastroduodenoscopy. In: Talley NJ, DeVault KR, Fleischer DE. Editors. Practical Gastroenterology and Hepatology Esophagus and Stomach $1^{\text {st }}$ Edition. Oxford: Blackwell Publishing, 2010. P 44-49.

3. Søreide K, Thorsen K, Harrison E, et al. Perforated peptic ulcer. Lancet 2015;386:1288-1298.

4. Baker S. Unenhanced helical CT versus plain abdominal radiography: A dissenting opinion. Radiology 1997;205: 45-47.

5. Hefny A, Abu-Zidan F. Sonographic diagnosis of intraperitoneal free air. J Emerg Trauma Shock 2011;4:511-513.

6. Marshall G. The cupola sign. Radiology 2006;241:623-624.

7. Levine M, Scheiner, Rubesin S, et al. Diagnosis of pneumoperitoneum on supine abdominal radiographs. AJR Am J Roentogenol 1991;156:731-735.

8. Sureka B, Bansal K, Arora A. Pneumoperitoneum: What to look for in a radiograph? J Family Med Prim Care. 2015; 4:477-8.

9. Ocampo Chaparro JM, García Mazuera K, Reynolds JW, et al. Abdomen Due to Cecal Volvulus Associated With Chilaiditi Syndrome. ACG Case Rep J. 2015;3:15-6.

10. Chertoff J, Khullar V, Burke L. Duodenal perforation following esophagogastroduodenoscopy (EGD) with cautery and epinephrine injection for peptic ulcer disease: An interesting case of nonoperative management in the medical intensive care unit (MICU). Int J Surg Case Rep. 2015;10:121-5. 